\title{
Effects of Amino Acids on Substrate Selection, Anaplerosis, and Left Ventricular Function in the Ischemic Reperfused Rat Heart
}

\author{
Michael E. Jessen, ${ }^{\star \|}$ Thomas E. Kovarik," F. Mark Jeffrey, ${ }^{\star}$ A. Dean Sherry, ${ }^{\star \Uparrow}$ Charles J. Storey, ${ }^{\star}$ \\ Robert Y. Chao," W. Steves Ring," and Craig R. Malloy *\$s \\ *Dallas Veterans Affairs Medical Center; ${ }^{\ddagger}$ Department of Radiology, Mary Nell and Ralph B. Rogers Magnetic Resonance Center, \\ ${ }^{\S}$ Division of Cardiology, Department of Internal Medicine; and "Division of Thoracic and Cardiovascular Surgery, \\ Department of Surgery, University of Texas Southwestern Medical Center, Dallas, Texas 75235-9085; \\ and 'Department of Chemistry, University of Texas at Dallas, Richardson, Texas 75083-0688
}

\begin{abstract}
The effect of aspartate and glutamate on myocardial function during reperfusion is controversial. A beneficial effect has been attributed to altered delivery of carbon into the citric acid cycle via substrate oxidation or by stimulation of anaplerosis, but these hypotheses have not been directly tested. ${ }^{13} \mathrm{C}$ isotopomer analysis is well suited to the study of myocardial metabolism, particularly where isotopic and metabolic steady state cannot be established. This technique was used to evaluate the effects of aspartate and glutamate (amino acids, AA) on anaplerosis and substrate selection in the isolated rat heart after $25 \mathrm{~min}$ of ischemia followed by $\mathbf{3 0}$ or $\mathbf{4 5} \mathrm{min}$ of reperfusion. Five groups of hearts $(n=8)$ provided with a mixture of $\left[1,2-{ }^{13} \mathrm{C}\right]$ acetate, $\left[3-{ }^{13} \mathrm{C}\right]$ lactate, and unlabeled glucose were studied: control, control plus AA, ischemia followed by 30 min of reperfusion, ischemia plus AA followed by 30 min of reperfusion, and ischemia followed by $45 \mathrm{~min}$ of reperfusion. The contribution of lactate to acetyl-CoA was decreased in postischemic myocardium (with a significant increase in acetate), and anaplerosis was stimulated. Metabolism of ${ }^{13} \mathrm{C}$-labeled aspartate or glutamate could not be detected, however, and there was no effect of AA on functional recovery, substrate selection, or anaplerosis. Thus, in contrast to earlier reports, aspartate and glutamate have no effect on either functional recovery from ischemia or on metabolic pathways feeding the citric acid cycle. (J. Clin. Invest. 1993. 92:831-839.) Key words: nuclear magnetic resonance $\bullet$ citric acid cycle metabolism $\bullet$ myocardium $\bullet$ ischemia aspartate $\bullet$ glutamate
\end{abstract}

\section{Introduction}

The activities of the metabolic pathways feeding the citric acid cycle are disrupted during ischemia and reperfusion (1-6). An understanding of the quantitative extent of these alterations is important for both diagnosis and therapy in reperfused myocardium. For example, clearance of ${ }^{11} \mathrm{C}$-enriched acetate may

Address correspondence to Michael E. Jessen, Department of Surgery, Division of Thoracic and Cardiovascular Surgery, University of Texas Southwestern Medical Center, 5323 Harry Hines Blvd., Dallas, TX 75235-8879.

Presented in part at the American Heart Association's 65th Scientific Sessions, November 17, 1992, New Orleans, LA.

Received for publication 4 January 1993 and in revised form 26 February 1993.

The Journal of Clinical Investigation, Inc.

Volume 92, August 1993, 831-839 be monitored in patients by positron tomography (7-9), yet the biochemical basis of altered tracer clearance during ischemia is incompletely understood (10-12). Of perhaps greater significance is the potential for improving recovery after ischemia by deliberately modifying carbon flow into the citric acid cycle. For example, a protective effect of aspartate and glutamate in models of myocardial ischemia is widely recognized (13-17) and application in clinical trials is underway $(18,19)$. Under normal conditions, amino acids contribute little to acetyl-CoA, but, in post-ischemic myocardium, aspartate and glutamate may indirectly alter substrate selection by stimulating both pyruvate transamination and glycolysis (20). Any shift from fatty acids to carbohydrates would be beneficial because of oxygen sparing, which has been documented in animal models (21-23) and supported by clinical observations (24-27).

Another potentially beneficial intervention is stimulation of pathways that replenish citric acid cycle intermediates. These pathways, termed anaplerosis by Kornberg (28), are active in the heart during ischemia (29), and stimulation with propionyl-carnitine improves recovery of ischemic myocardium (30). Aspartate and glutamate may enter the citric acid cycle via transamination and increase the concentration of citric acid cycle intermediates (15). Activation of these pathways may be beneficial by increasing net flux through the citric acid cycle or by increasing high energy phosphates via substratelevel phosphorylation (31).

Although substrate selection in reperfused myocardium has been studied extensively, information concerning anaplerotic activity is not available. Substrate selection is usually determined by measurements of arteriovenous differences or ${ }^{14} \mathrm{CO}_{2}$ release from ${ }^{14} \mathrm{C}$-enriched compounds, and measurement of anaplerosis typically involves purification and carbon-by-carbon degradation of intermediates (32). Both measurements are difficult in reperfused myocardium where isotopic and metabolic steady state are difficult to establish. ${ }^{13} \mathrm{C}$ is a stable isotope that has recently been applied to numerous problems in intermediary metabolism $(33-35) .{ }^{13} \mathrm{C}$ isotopomer analysis offers advantages over other measurements of substrate selection because it is technically simple, and anaplerosis and competition among several substrates can be assessed in a single experiment (36-38). Further, requirement for steady state conditions does not apply to measurements of substrate selection $(39,40)$.

For these reasons ${ }^{13} \mathrm{C}$-nuclear magnetic resonance (NMR) spectroscopy was used to address two questions: what are the

1. Abbreviations used in this paper: AA, amino acids; LV, left ventricular; NMR, nuclear magnetic resonance. 
effects of prolonged ischemia followed by reperfusion on substrate selection and anaplerosis? Are the protective effects of glutamate and aspartate associated with altered substrate selection, anaplerosis, or both? Prolonged ischemia was studied because earlier observations indicate little effect of brief ischemia on substrate selection or functional recovery (40). The metabolic effects of these amino acids are of longstanding interest, and plausible biochemical mechanisms that are consistent with their beneficial effects have been suggested but not tested. Lactate was examined because its utilization is avid in normoxic myocardium (41) but is suppressed during severe ischemia because of inhibition of the pyruvate dehydrogenase complex (42). Acetate was studied because it provides an alternative carbon source, which bypasses pyruvate dehydrogenase, and because of its importance as a tracer for positron tomography studies.

\section{Methods}

Materials. The following ${ }^{13} \mathrm{C}$-enriched compounds were used: $\left[3-{ }^{13} \mathrm{C}\right]-$ sodium L-lactate, $\left[3-{ }^{13} \mathrm{C}\right]$ sodium-D,L-aspartate ( 99 and $94 \%$, respectively; MSD Isotopes, Montreal, Quebec, Canada), $\left[1,2-{ }^{13} \mathrm{C}\right]$ sodium acetate (99\%; Isotec Inc., Miamisburg, $\mathrm{OH})$, and $\left[\mathrm{U}-{ }^{13} \mathrm{C}\right] \mathrm{L}$-glutamic acid (98\%; Cambridge Isotope Laboratories, Inc., Woburn, MA).

Perfusion technique and hemodynamic monitoring. Male SpragueDawley rats weighing $300-400 \mathrm{~g}$ with free access to food and water were used in these investigations and were cared for in the Animal Resources Center (43). Animals were anesthetized with $4 \%$ chloral hydrate ( 1 $\mathrm{ml} / 100 \mathrm{~g}$, i.p. $)$, and heparin $(100 \mathrm{U} / 100 \mathrm{~g})$ was administered via the femoral vein. The heart was excised, and the aorta was cannulated for retrograde (Langendorff) perfusion. Hearts were perfused at $100 \mathrm{~cm}$ of water throughout the experiment except during the ischemic periods. Typical coronary flow was $15-18 \mathrm{ml} / \mathrm{min}$, and the perfusate was not recirculated. The heart chamber was immersed in a controlled-temperature bath maintained at $37^{\circ} \mathrm{C}$.

A latex balloon was inserted into the left atrium across the mitral valve into the left ventricle. This balloon was connected via tubing to a pressure transducer, and pressure and $\mathrm{dP} / \mathrm{dT}$ were recorded continuously. Pacing electrodes were attached to the right atrium and all hearts were paced at 300 beats per min at twice the diastolic threshold. Hearts that had an intrinsic rate $>300$ beats per min were not paced. For inclusion in this study, rat hearts were required to achieve a left ventricular (LV) peak pressure of $90 \mathrm{mmHg}$ at an end-diastolic pressure of $<10 \mathrm{mmHg}$ by the end of the 20 -min stability period. About $20 \%$ of hearts failed these criteria.

Coronary flow and functional data were recorded at the end of the stability period, at the end of the 25 -min period for hearts not rendered ischemic, and at 15 -min intervals during the reperfusion period. Developed pressure was calculated as the difference between LV peak and end-diastolic pressures. Heart rate was 300 beats per min for paced hearts and varied between 300 and 360 beats per min in nonpaced hearts. Rate-pressure product was calculated as the product of developed pressure and heart rate.

Perfusate composition. The perfusate used for these experiments was a modified Krebs-Henseleit solution bubbled with $95 \% \mathrm{O}_{2} / 5 \%$ $\mathrm{CO}_{2}$. For experiments done without amino acids, the perfusate contained the following $(\mathrm{mM}): 118.0 \mathrm{NaCl}, 4.7 \mathrm{KCl}, 1.2 \mathrm{MgSO}_{4}, 1.2$ $\mathrm{NaH}_{2} \mathrm{PO}_{4}, 25.0 \mathrm{NaHCO}_{3}, 1.2 \mathrm{CaCl}_{2}, 10.0$ glucose, 1.0 lactate, and 0.25 sodium acetate. Two groups (see below) were studied with both glutamate $(5 \mathrm{mM})$ and aspartate $(5 \mathrm{mM})$ in the perfusate. For these experiments, the perfusate was modified to maintain a constant sodium concentration and osmolality. Unlabeled glucose $(10 \mathrm{mM})$ was present throughout all experiments.

Each of the above perfusing solutions was made in two forms. In one, unlabeled lactate and acetate were used. In the other solution (used during the final 30 or $45 \mathrm{~min}$ of the experiment) $1 \mathrm{mM}\left[3-{ }^{13} \mathrm{C}\right]-$ lactate and $0.25 \mathrm{mM}\left[1,2-{ }^{13} \mathrm{C}\right]$ acetate were substituted in the perfusion solution. Each lot of lactate solution was assayed to determine concentration (44). All ${ }^{13} \mathrm{C}$-enriched compounds were checked for fractional enrichment by either ${ }^{13} \mathrm{C}$ or ${ }^{1} \mathrm{H}$-NMR spectroscopy to confirm the stated enrichment.

Experimental protocol. The experimental protocol is shown in Fig. 1 for five groups of eight animals. In group 1 (ischemia) rat hearts were perfused for a stabilization period of $20 \mathrm{~min}$, and then made ischemic for $25 \mathrm{~min}$ by clamping the aortic cannula. After completion of the ischemic period, hearts were reperfused for $30 \mathrm{~min}$ using the solution containing the ${ }^{13} \mathrm{C}$-enriched substrates and freeze-clamped. Group 2 (control) hearts were stabilized for $20 \mathrm{~min}$ and then perfused for an additional 25 -min interval. The perfusing solution was then switched to one containing the ${ }^{13} \mathrm{C}$-labeled substrates and after an additional 30 min the hearts were freeze-clamped.

Group 3 (ischemia plus amino acids) followed an identical protocol to group 1 with, the exception that the perfusing solutions contained $5 \mathrm{mM}$ glutamate and $5 \mathrm{mM}$ aspartate. Group 4 (control plus amino acids) followed an identical pattern to group 2 with the exception that the perfusating solutions contained glutamate and aspartate. The protocol for group 5 was identical to group 1 except that the hearts were reperfused for $45 \mathrm{~min}$ with labeled substrates.

Tissue extraction and ${ }^{13} C-N M R$ spectroscopy. Hearts were freezeclamped with aluminum tongs chilled in liquid nitrogen, extracted with cold perchloric acid, neutralized with $\mathrm{KOH}$, freeze-dried, and dissolved in $0.5 \mathrm{ml}$ of $\mathrm{D}_{2} \mathrm{O}$ for NMR analysis (36). Proton decoupled ${ }^{13} \mathrm{C}$ spectra were obtained at $100.3 \mathrm{MHz}$ on a spectrometer (Omega; General Electric Co., Wilmington, MA). Spectra of heart extracts were acquired in a $5-\mathrm{mm}$ tube using a $45^{\circ}$ carbon pulse, $16 \mathrm{~K}$ data points over $22,000-26,000 \mathrm{~Hz}$, and a 6-s delay between pulses. Protons were decoupled using the WALTZ sequence at two power levels (BILEV), and the temperature was maintained at $25^{\circ} \mathrm{C}$ using a standard variable temperature accessory (General Electric Co.). All spectra were zerofilled to $32 \mathrm{~K}$ points before Fourier transformation. The relative areas of the multiplet components in each glutamate resonance were determined using commercial software (NMR-1; New Methods Research, Inc., Syracuse, NY).

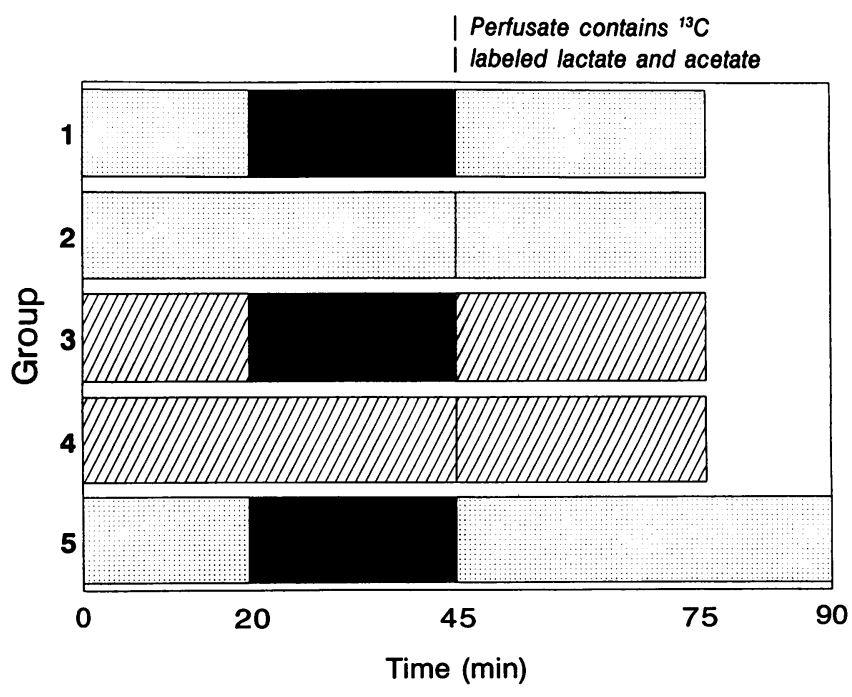

Figure 1. Experimental protocol. Aspartate and glutamate were present in the perfusate throughout the experiment in groups 3 and 4 (shaded). Groups 1, 3, and 5 were ischemic for $25 \mathrm{~min}$ (black), followed by reperfusion. All hearts were supplied with ${ }^{13} \mathrm{C}$-enriched substrates after $45 \mathrm{~min}$. Groups 1-4 were freeze clamped $75 \mathrm{~min}$ after the initiation of perfusion, and group 5 was freeze clamped at $90 \mathrm{~min}$. 


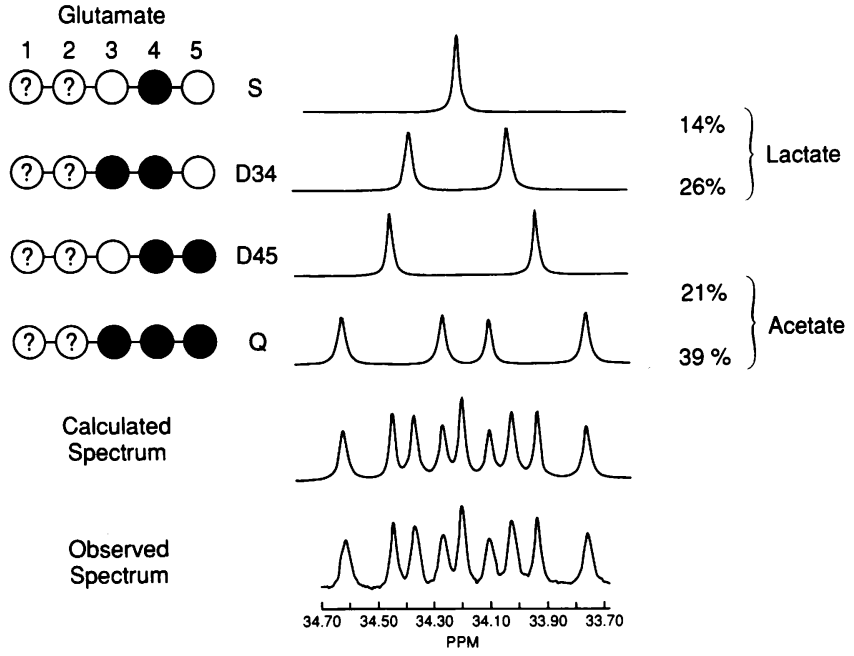

Figure 2. Analysis of the $\mathrm{C} 4$ resonance of glutamate. A proton-decoupled ${ }^{13} \mathrm{C}$-NMR spectrum of glutamate $\mathrm{C} 4$ is shown in the lower panel. This observed spectrum can be resolved into four multiplets, shown above. The relative intensities of these multiplets is proportional to the relative concentration of the labeling patterns in the glutamate pools, shown at the left where filled circles represent ${ }^{13} \mathrm{C}$, open circles represent ${ }^{12} \mathrm{C}$, and ? indicates that labeling does not influence analysis of the $\mathrm{C} 4$. The contribution of $\left[1,2{ }^{13} \mathrm{C}\right]$ acetate relative to $\left[3-{ }^{13} \mathrm{C}\right]$ lactate is simply the ratio $(\mathrm{D} 45+\mathrm{Q}) /(\mathrm{S}+\mathrm{D} 34)$ or 1.5 in this example.

Analysis of ${ }^{13} C-N M R$ spectra. Under the conditions reported here, ${ }^{13} \mathrm{C}$ continuously enters the citric acid cycle via citrate synthase. Intermediates of the cycle become enriched in multiple sites as a consequence of turnover of citric acid cycle intermediates. Ultimately, multiplets due to ${ }^{13} \mathrm{C}-{ }^{13} \mathrm{C}$ coupling are observed in the ${ }^{13} \mathrm{C}$ spectrum of glutamate, and the relative intensities of the multiplets may be analyzed to determine the fraction of acetyl-CoA derived from exogenous substrates. A simple example is shown in Fig. $2 \cdot\left[2-{ }^{13} \mathrm{C}\right]$ acetyl-CoA can only be derived from $\left[3-{ }^{13} \mathrm{C}\right]$ lactate, and $\left[1,2-{ }^{13} \mathrm{C}\right]$ acetyl-CoA can only be derived from $\left[1,2-{ }^{13} \mathrm{C}\right]$ acetate. These two labeling patterns in acetyl$\mathrm{CoA}$ result in characteristic labeling patterns in the 4 carbon (C4) of glutamate. As shown in Fig. 2, competition between these substrates may be appreciated by simple inspection of the $C 4$ resonance $(40)$.

The glutamate spectrum was quantitatively analyzed using the nonsteady state $(37,39)$ and steady state (36-39) methods. The nonsteady state analysis measures the contribution of ${ }^{13} \mathrm{C}$-enriched lactate, acetate, and unlabeled sources to acetyl-CoA. It is based upon the information in the multiplets of the glutamate $\mathrm{C} 4$ resonance described above plus the ratio of enrichment in glutamate $\mathrm{C} 3$ and $\mathrm{C} 4$. Because these multiplets represent the patterns of ${ }^{13} \mathrm{C}$ and ${ }^{12} \mathrm{C}$ in glutamate, the analysis is equally sensitive to unlabeled as well as labeled carbon. Assumptions about metabolic and isotopic steady state are not required $(37,39)$.

The steady state analysis provides a complete description of the labeling pattern in the acetyl-CoA pool, including a measure of relative flux through the anaplerotic pathways. This analysis requires input of all multiplet components in glutamate $\mathrm{C} 2, \mathrm{C} 3$, and $\mathrm{C} 4$ and requires that the system be in metabolic and isotopic steady state.

Statistical analysis. Statistical comparisons were performed using commercially available statistical software (45). Comparison of functional parameters at different time points was performed using a repeated-measures analysis of variance. Comparison of metabolic results was performed using a one-way analysis of variance with StudentNewman-Keuls test for differences between groups ( $\alpha$ was set at 0.05 ). All results are reported as mean $\pm \mathrm{SD} ; n=8$ for each group.

\section{Results}

Myocardial performance was constant throughout the experimental period in normoxic hearts (groups 2 and 4), as anticipated, whereas LV performance was significantly impaired during the reperfusion period in ischemic hearts (groups 1, 3, and 5). Functional observations before ischemia and at $15-\mathrm{min}$ intervals during reperfusion are presented in Table $\mathrm{I}$.

A typical ${ }^{13} \mathrm{C}-\mathrm{NMR}$ spectrum from a control heart (group 2 ) is shown in Fig. 3. All glutamate carbons become ${ }^{13} \mathrm{C}$ enriched and extensive spin-spin coupling is observed in each carbon resonance. The ${ }^{13} \mathrm{C}$-NMR spectra from postischemic hearts differed significantly compared with those obtained from control hearts. Representative examples of the carbon spectra from the $\mathrm{C} 2, \mathrm{C} 3$, and $\mathrm{C} 4$ carbon positions of glutamate in one heart subjected to ischemia (group 1) and one control heart (group 2) are shown in Fig. 4. The multiplets observed in the ${ }^{13} \mathrm{C}$-NMR spectra are summarized in Table II, and the contributions of lactate, acetate, and unlabeled substrates to acetylCoA are shown in Table III.

Effect of ischemia on substrate selection and anaplerosis. The glutamate multiplets arising from labeled acetate will be detected in the C4 resonance as a doublet (D45) or quartet (Q). On the other hand, multiplets arising from labeled lactate will be detected as a singlet (S) or doublet (D34). With this information the $\mathrm{C} 4$ multiplets in normoxic and postischemic hearts can be easily compared and interpreted: the oxidation of labeled acetate increased relative to the oxidation of labeled lactate (Fig. 4 and Table II).

Sources of acetyl-CoA were measured by analysis of the ${ }^{13} \mathrm{C}$ spectra assuming either steady state or nonsteady state metabolic conditions ( Table III and Fig. 5). Either method provides a complete description of the acetyl-CoA-labeling patterns, including unlabeled acetyl-CoA. Because the labeling of lactate and acetate are known, the sources of acetyl-CoA (including unlabeled sources) are also determined. Results were compared for all five groups by analysis of variance ( Table III). The contributions to acetyl-CoA in groups 1,3 , and 5 were not different. Similarly, sources of acetyl-CoA were not different in groups 2 and 4 . However, groups 1,3 , and 5 (ischemic hearts) were significantly different compared with groups 2 and 4 (normoxic hearts). Thus, ischemia but not amino acids significantly shifted substrate preference toward acetate. Statistical analysis of substrate selection measured by the steady state method showed the same pattern: the lactate contribution was lower and acetate contribution was significantly greater in the ischemic groups ( 1,3 , and 5$)$ compared with control groups ( 2 and 4). The contribution of unlabeled sources (glucose in the perfusate, glycogen, and triglycerides) was also slightly higher in reperfused hearts.

A comparison of substrate utilization as measured using the nonsteady state method in hearts made ischemic and reperfused for 30 min (group 1) versus hearts made ischemic and reperfused for 45 min (group 5) indicates that equivalent results were obtained for each reperfusion period (lactate contribution, $0.29 \pm 0.07$ vs. $0.34 \pm 0.08$ and acetate contribution, $0.42 \pm 0.08$ vs. $0.40 \pm 0.07$, for groups 1 vs. 5 , respectively). This indicates that the tissue was in isotopic steady state after $30 \mathrm{~min}$ of reperfusion, thus allowing the application of published steady state methods to estimate anaplerotic flux in ischemic versus control tissue. Anaplerotic flux (Table IV) was higher in all ischemic groups $(1,3$, and 5) compared with normoxic 


\begin{tabular}{|c|c|c|c|c|}
\hline Group & $\begin{array}{c}20 \text { min } \\
\text { (baseline) }\end{array}$ & $\begin{array}{c}60 \mathrm{~min} \\
(15 \mathrm{~s} \mathrm{rep})\end{array}$ & $\begin{array}{c}75 \mathrm{~min} \\
(30 \mathrm{~s} \text { rep) }\end{array}$ & $\begin{array}{c}90 \mathrm{~min} \\
\text { (45 s rep) }\end{array}$ \\
\hline \multicolumn{5}{|l|}{ Developed pressure } \\
\hline 1 isc - & $93 \pm 8$ & $23 \pm 15^{*}$ & $28 \pm 17^{*}$ & \\
\hline 2 nor - & $94 \pm 9$ & $98 \pm 7$ & $102 \pm 14$ & \\
\hline 3 isc + & $94 \pm 10$ & $21 \pm 12^{*}$ & $34 \pm 16^{*}$ & \\
\hline 4 nor + & $90 \pm 11$ & $99 \pm 10$ & $100 \pm 9$ & \\
\hline 5 isc - & $98 \pm 16$ & $30 \pm 19 *$ & $41 \pm 23^{*}$ & $45 \pm 24$ \\
\hline \multicolumn{5}{|l|}{ Peak positive $\mathrm{dP} / \mathrm{dT}$} \\
\hline 1 isc - & $2144 \pm 446$ & $488 \pm 349^{*}$ & $668 \pm 496^{*}$ & \\
\hline 2 nor - & $2259 \pm 370$ & $2470 \pm 370$ & $2463 \pm 423$ & \\
\hline 3 isc + & $2188 \pm 247$ & $401 \pm 244^{*}$ & $650 \pm 369^{*}$ & \\
\hline 4 nor + & $2085 \pm 259$ & $2409 \pm 297$ & $2463 \pm 283$ & \\
\hline 5 isc - & $2194 \pm 498$ & $613 \pm 363^{*}$ & $888 \pm 496^{*}$ & $1020 \pm 556$ \\
\hline \multicolumn{5}{|c|}{ Rate pressure production $\left(\times 10^{3}\right)$} \\
\hline 1 isc - & $29.5 \pm 3.6$ & $7.0 \pm 4.8^{*}$ & $9.1 \pm 5.8^{*}$ & \\
\hline 2 nor - & $28.4 \pm 2.4$ & $30.2 \pm 2.4$ & $31.4 \pm 5.1$ & \\
\hline 3 isc + & $28.7 \pm 3.1$ & $6.4 \pm 3.6^{*}$ & $10.5 \pm 4.7^{*}$ & \\
\hline 4 nor + & $27.1 \pm 3.2$ & $30.0 \pm 3.2$ & $30.4 \pm 2.7$ & \\
\hline 5 isc - & $30.1 \pm 4.7$ & $9.1 \pm 5.6^{*}$ & $12.3 \pm 6.9^{*}$ & $13.4 \pm 7.1$ \\
\hline \multicolumn{5}{|l|}{ Coronary flow } \\
\hline 1 isc - & $17 \pm 6$ & $12 \pm 2$ & $11 \pm 3$ & \\
\hline 2 nor - & $17 \pm 4$ & $18 \pm 4$ & $18 \pm 5$ & \\
\hline 3 isc + & $19 \pm 2$ & $17 \pm 8$ & $19 \pm 6$ & \\
\hline 4 nor + & $15 \pm 5$ & $20 \pm 6$ & $18 \pm 5$ & \\
\hline 5 isc - & $14 \pm 2$ & $11 \pm 4$ & $10 \pm 3$ & $9 \pm 3$ \\
\hline
\end{tabular}

Developed pressure, peak positive $\mathrm{dP} / \mathrm{dT}$, and rate-pressure product were measured at selected intervals after initiation of perfusion. Time after reperfusion is relevant to groups 1,3 , and 5 . Developed pressure is reported in $\mathrm{mmHg}$; peak positive $\mathrm{dP} / \mathrm{dT}$ is in $\mathrm{mmHg} / \mathrm{s}$, rate-pressure product is in $\mathrm{mm} \mathrm{Hg} \times$ beats per min, and coronary flow is in $\mathrm{ml} / \mathrm{min}$. Results are mean $\pm \mathrm{SD}(n=8)$. Isc, ischemic and reperfused; nor, normoxic; + , glutamate and aspartate in the perfusate; - , glutamate and aspartate not present in the perfusate.

* Different from groups 2 and 4 at that time, repeated measures analysis of variance.

hearts (groups 2 and 4 ). In all cases, the source of anaplerosis appeared to arise mainly $(\sim 80 \%)$ from unlabeled carbon sources.

Effect of aspartate and glutamate on ventricular performance and metabolism. Aspartate and glutamate had no effect on myocardial function in normoxic hearts (Table I) nor were any differences in functional recovery observed after ischemia between hearts reperfused in the presence (group 3 ) or absence (group 1 ) of glutamate and aspartate. Near the end of the ischemic period, increases in diastolic pressure were observed. The time to increased diastolic pressure, defined as a rise of $4 \mathrm{mmHg}$, was $16.4 \pm 1.5 \mathrm{~min}$ in control hearts (group 1, mean $\pm \mathrm{SD}$ ) and $16.6 \pm 1.2 \mathrm{~min}$ in hearts exposed to glutamate and aspartate (group 3 ). Thus, earlier observations of a protective effect were not confirmed.

Aspartate and glutamate also had no effect on the sources of acetyl-CoA in normoxic myocardium (Table III, group 2 vs. 4). In both cases, lactate was the predominant source, and unlabeled substrates provided only $11-14 \%$ of acetyl-CoA. Although ischemia significantly altered substrate selection (group 1 vs. 2 , discussed above), aspartate and glutamate again had no effect on substrate selection after ischemia compared with untreated hearts (group 1 vs. 3). Also, anaplerotic flux was low in normoxic hearts and was not altered by aspartate and glutamate (Table IV). Reperfusion after $25 \mathrm{~min}$ of ischemia increased anaplerosis relative to citric acid cycle flux (Table IV), but this increase was not affected by amino acids in the perfusate. Thus, these amino acids did not appear to alter either oxidative or anaplerotic pathways feeding the citric acid cycle.

Direct detection of aspartate and glutamate metabolism. Although an effect of glutamate and aspartate on anaplerosis or substrate selection could not be detected in ischemic hearts, these results do not exclude utilization of exogenous glutamate (or aspartate) in anaplerotic reactions that were stimulated by ischemia. To test this possibility, the ischemic and normoxic protocols with amino acids (groups 3 and 4 ) were repeated in the presence of $5 \mathrm{mM}\left[\mathrm{U}^{1{ }^{13}} \mathrm{C}\right] \mathrm{L}$-glutamate, $5 \mathrm{mM}$ unlabeled aspartate, and with unlabeled acetate and lactate. Thus, the only source of ${ }^{13} \mathrm{C}$ in these experiments is exogenous glutamate. Metabolism in the citric acid cycle must yield $\left[1,2,3-{ }^{13} \mathrm{C}\right] \alpha-$ ketoglutarate and $\left[1,2,3-{ }^{13} \mathrm{C}\right]$ glutamate. This coupling pattern, easily distinguished from $\left[\mathrm{U}_{-1}^{13} \mathrm{C}\right]$ glutamate, was not detected in either ischemic or control hearts. Entry of aspartate was studied in a similar protocol using $\left[3-{ }^{13} \mathrm{C}\right]$ aspartate (other compounds were unlabeled). Again, the metabolic products of this compound could not be detected in tissue extracts. To- 

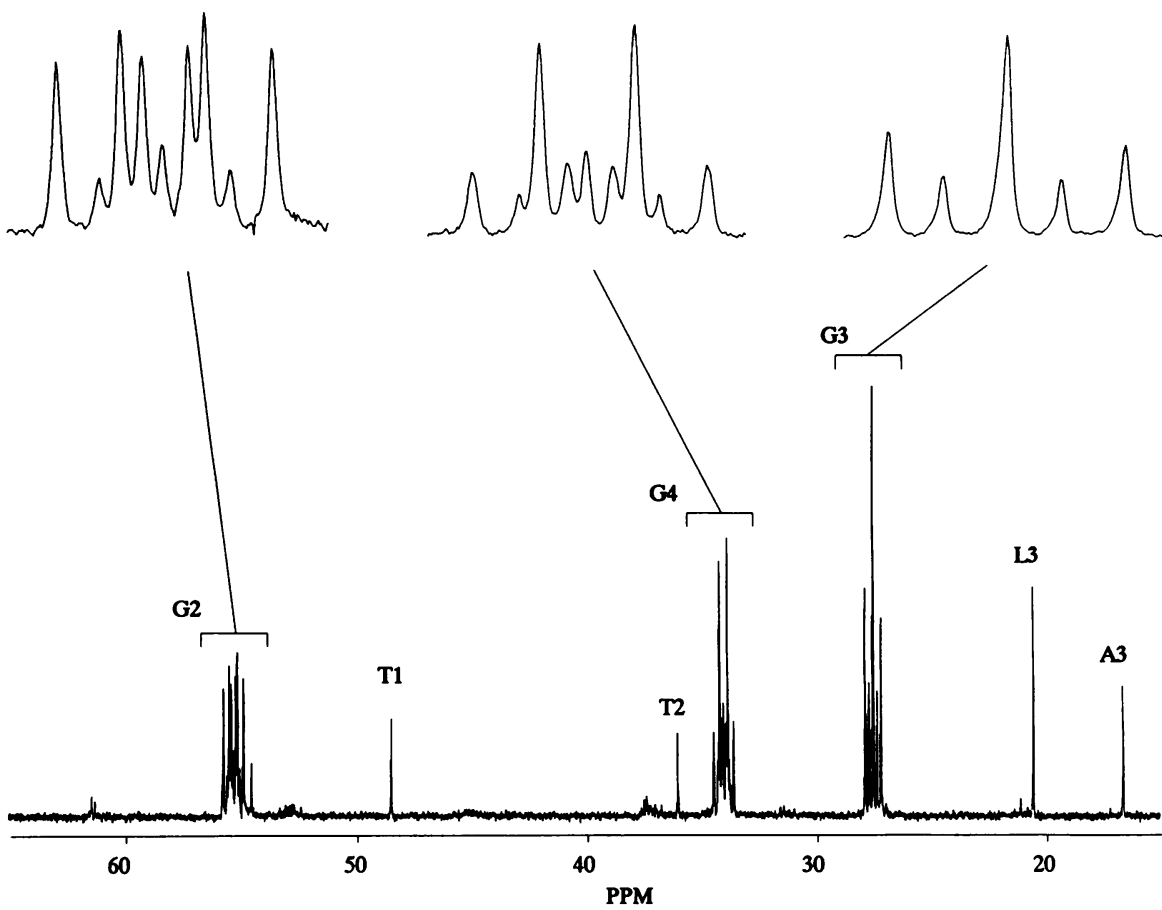

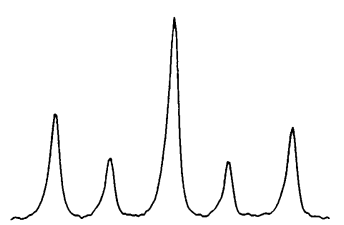

Figure 3. Proton-decoupled ${ }^{13} \mathrm{C}-\mathrm{NMR}$ spectrum of an extract of a normoxic heart not supplied with amino acids. Although glutamate resonances dominate the spectrum, other metabolites can be detected. $A 3$, alanine $\mathrm{C} 3 ; L 3$, lactate $\mathrm{C} 3 ; G 2$, glutamate $\mathrm{C} 2 ; G 3$, glutamate $\mathrm{C} 3$; $G 4$, glutamate $\mathrm{C} 4 ; T 1$ and $T 2$, taurine $\mathrm{C} 1$ and $\mathrm{C} 2$. gether, these observations confirm that neither aspartate nor glutamate are metabolized in the citric acid cycle under these conditions.

Correlation between functional recovery and metabolic state. To examine the relationship between myocardial performance and metabolic state, data from all 40 hearts were combined. A strong correlation was observed between cardiac function and pathways feeding the citric acid cycle (Fig. 6). There was a positive correlation between lactate utilization and ratepressure product ( $r=0.92$, Fig. $6 A$ ). A similar correlation was observed between the ratio of lactate to acetate utilization and ventricular function ( $r=0.91$, Fig. $6 \mathrm{~B})$. A negative correlation was found between anaplerosis and function $(r=-0.90$, Fig. 6 $C)$. Thus, higher levels of anaplerosis relative to citric acid cycle flux were associated with impaired ventricular performance. These relations remained statistically significant when only postischemic hearts were analyzed.

\section{Discussion}

This model of prolonged global normothermic ischemia and reperfusion was chosen to allow comparison to numerous previous investigations $(14,16,17,20,31,46) .{ }^{13} \mathrm{C}-\mathrm{NMR}$ was used to test previous hypotheses regarding the effects of amino acids on substrate selection, anaplerosis, and functional recovery after ischemia.

The protective effects of glutamate and aspartate on ischemic heart tissue were not confirmed in this study. Other investigators have observed improvements in postischemic cardiac performance when the perfusate is enriched with these amino acids. Exposure to $2 \mathrm{mM}$ glutamate before, during, and after severe ischemia leads to improved functional recovery in the intact rabbit heart (16). Glutamate at higher concentrations (20 mM) during underperfusion of isolated rat hearts greatly reduced the resulting contractile dysfunction (14). Addition of
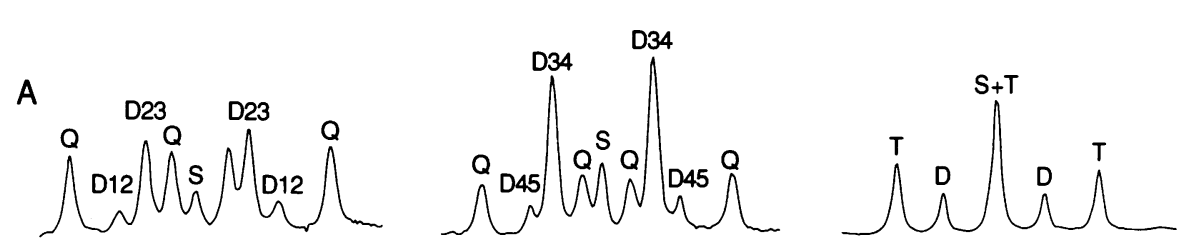

Figure $4 .{ }^{13} \mathrm{C}-\mathrm{NMR}$ spectra of the $\mathrm{C} 2, \mathrm{C} 3$, and $\mathrm{C} 4$ carbons of glutamate from heart extracts. A spectrum from a nonischemic heart (group 2) is shown in $A$. In the C4 resonance from the ischemic heart (group $1, B)$, the multiplets due to acetate ( $D 45$
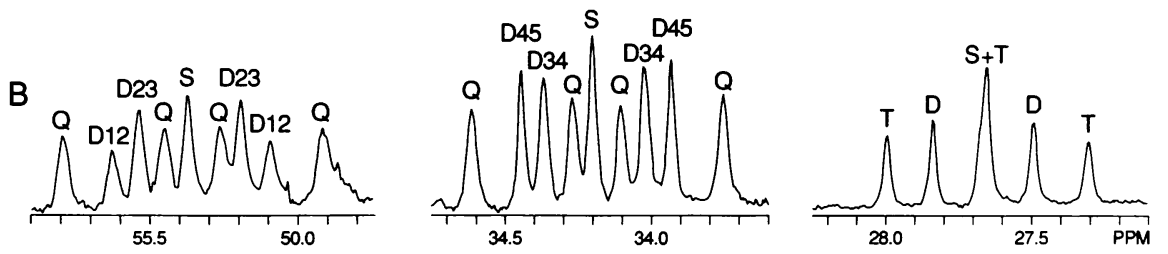
and $Q$ ) are substantially increased relative to those due to lactate ( $S$ and $D 34$ ), which indicates increased preference for acetate relative to lactate in reperfused myocardium. $S$, singlet; $D$, doublet; $T$, triplet; $Q$, quartet. 
Table II. Multiplet Areas in the ${ }^{13}$ C-NMR Spectrum

\begin{tabular}{|c|c|c|c|c|c|c|c|c|c|c|c|}
\hline \multirow[b]{2}{*}{ Group } & \multicolumn{4}{|c|}{$\mathrm{C} 2$} & \multicolumn{3}{|c|}{$\mathrm{C} 3$} & \multicolumn{4}{|c|}{ C4 } \\
\hline & $\mathbf{S}$ & D23 & D12 & $\mathbf{Q}$ & $\mathbf{S}$ & D & $\mathbf{T}$ & $S$ & D34 & D45 & $\mathbf{Q}$ \\
\hline 1 isc - & $0.17 \pm 0.03$ & $0.26 \pm 0.03$ & $0.19 \pm 0.03$ & $0.37 \pm 0.04$ & $0.12 \pm 0.04$ & $0.40 \pm 0.04$ & $0.48 \pm 0.07$ & $0.16 \pm 0.03$ & $0.24 \pm 0.06$ & $0.25 \pm 0.04$ & $0.34 \pm 0.05$ \\
\hline 2 nor - & $0.08 \pm 0.04$ & $0.34 \pm 0.02$ & $0.09 \pm 0.03$ & $0.48 \pm 0.08$ & $0.03 \pm 0.02$ & $0.25 \pm 0.08$ & $0.71 \pm 0.10$ & $0.12 \pm 0.05$ & $0.51 \pm 0.04$ & $0.08 \pm 0.02$ & $0.29 \pm 0.07$ \\
\hline 3 isc + & $0.17 \pm 0.03$ & $0.28 \pm 0.02$ & $0.14 \pm 0.04$ & $0.41 \pm 0.06$ & $0.11 \pm 0.02$ & $0.34 \pm 0.05$ & $0.56 \pm 0.06$ & $0.18 \pm 0.02$ & $0.29 \pm 0.04$ & $0.19 \pm 0.03$ & $0.33 \pm 0.06$ \\
\hline 4 nor + & $0.07 \pm 0.02$ & $0.34 \pm 0.02$ & $0.07 \pm 0.01$ & $0.53 \pm 0.03$ & $0.02 \pm 0.01$ & $0.20 \pm 0.03$ & $0.77 \pm 0.03$ & $0.10 \pm 0.02$ & $0.52 \pm 0.03$ & $0.07 \pm 0.01$ & $0.31 \pm 0.04$ \\
\hline 5 isc - & $0.13 \pm 0.03$ & $0.27 \pm 0.03$ & $0.16 \pm 0.02$ & $0.44 \pm 0.03$ & $0.08 \pm 0.04$ & $0.35 \pm 0.03$ & $0.57 \pm 0.07$ & $0.16 \pm 0.02$ & $0.30 \pm 0.08$ & $0.19 \pm 0.04$ & $0.35 \pm 0.04$ \\
\hline
\end{tabular}

$\mathrm{C} 2, \mathrm{C} 3$, and $\mathrm{C} 4$ refer to the carbon resonances observed in the proton-decoupled ${ }^{13} \mathrm{C}-\mathrm{NMR}$ spectrum of glutamate $(37,39)$. Each resonance is composed of multiplets which are denoted as $S$, singlet, $D$, doublet (with carbon-carbon coupling); $T$, triplet; and $\mathrm{Q}$, quartet, or doublet of doublets. Examples of ${ }^{13} \mathrm{C}$ spectra are shown in Fig. 4, and other abbreviations are in Table I. Results are mean $\pm \operatorname{SD}(n=8)$.

aspartate and glutamate to cardioplegic solutions during (17) or after (47) ischemia also has been associated with superior recovery of function during the reperfusion period.

It is difficult to attribute the lack of functional benefit of aspartate and glutamate found in this study to major differences in the experimental model compared with earlier studies. The concentrations of amino acids used in the present study were higher than those found effective in some earlier reports (16). In our study, amino acids were provided both before and after ischemia to increase the chance of showing an effect. The severity of the ischemic episode may have been too great, although beneficial effects have been observed with longer periods of low flow ischemia $(14,31)$. Similar to our results, Galinanes et al. (46) could not detect a protective effect independent of changes in perfusate sodium caused by addition of sodium aspartate. Nevertheless, the effects of these amino acids may be highly dependent on the experimental protocol, and a salutary effect of exogenous amino acids may certainly be present under other conditions.

The functional benefits described earlier have been attributed to several mechanisms. One suggestion is that glutamate may alter myocardial substrate utilization. Thomassen and coworkers (48) demonstrated a change in substrate preference from free fatty acid use toward glucose and glutamate consumption in patients receiving an infusion of glutamate. Pacing-induced myocardial lactate release was reduced after glutamate administration in patients with ischemic heart disease, and pacing tolerance, an indirect indication of ischemic threshold, was improved (49). We found no alteration in substrate selection upon addition of $5 \mathrm{mM}$ aspartate and glutamate in control hearts or in hearts recovering from a 25 -min ischemic episode.

Another potential protective mechanism for these amino acids could involve their entry into the citric acid cycle intermediate pools via transamination of glutamate and pyruvate to yield $\alpha$-ketoglutarate plus alanine or between aspartate and pyruvate to yield oxaloacetate plus alanine. During ischemia the concentration of alanine increases (29) and the concentrations of glutamate and aspartate decrease, perhaps indicating that the myocardium is capable of transamination via these pathways. Addition of exogenous glutamate could theoretically increase $\alpha$-ketoglutarate levels (16), which could then generate high energy phosphates via substrate level phosphorylation (14) in subsequent reactions. However, succinyl-CoA generation from $\alpha$-ketoglutarate requires $\mathrm{NAD}^{+}$, which is depleted during ischemia. Some authors have suggested that oxaloacetate derived from aspartate may oxidize NADH via reversal of malate dehydrogenase and therefore support a greater role for aspartate than glutamate in ischemic protection (17). Furthermore, others have demonstrated a direct inhibitory effect of glutamate on pyruvate carboxylase $(50)$ in other tissues, which is thought to be a major anaplerotic mechanism in the heart (51).

Despite the absence of functional benefits, the metabolic changes suggested by others should have been readily detected in the present study because ${ }^{13} \mathrm{C}-\mathrm{NMR}$ is sensitive to the activity of pathways feeding acetyl-CoA and entry of either labeled or unlabeled molecules into the citric acid cycle pools via an

Table III. Sources of Acetyl-CoA

\begin{tabular}{|c|c|c|c|c|c|c|}
\hline \multirow[b]{2}{*}{ Group } & \multicolumn{3}{|c|}{ Nonsteady state analysis } & \multicolumn{3}{|c|}{ Steady state analysis } \\
\hline & lactate & acetate & unlabeled & lactate & acetate & unlabeled \\
\hline 1, isc - & $0.29 \pm .07$ & $0.42 \pm .08$ & $0.29 \pm .07$ & $0.31 \pm .06$ & $0.44 \pm .06$ & $0.25 \pm .06$ \\
\hline 2, nor - & $0.55 \pm .05$ & $0.31 \pm .07$ & $0.14 \pm .07$ & $0.55 \pm .03$ & $0.31 \pm .07$ & $0.14 \pm .06$ \\
\hline 3, isc + & $0.35 \pm .04$ & $0.40 \pm .07$ & $0.26 \pm .06$ & $0.39 \pm .04$ & $0.43 \pm .07$ & $0.18 \pm .05$ \\
\hline 4, nor + & $0.56 \pm .04$ & $0.33 \pm .04$ & $0.11 \pm .03$ & $0.56 \pm .04$ & $0.34 \pm .04$ & $0.10 \pm .02$ \\
\hline 5, isc - & $0.34 \pm .08$ & $0.40 \pm .07$ & $0.27 \pm .04$ & $0.37 \pm .08$ & $0.43 \pm .05$ & $0.20 \pm .05$ \\
\hline
\end{tabular}

${ }^{13} \mathrm{C}-\mathrm{NMR}$ spectra were analyzed using the steady state and nonsteady state analysis as described in the text. Results are mean $\pm \mathrm{SD}(n=8)$. See Table I for other abbreviations. 


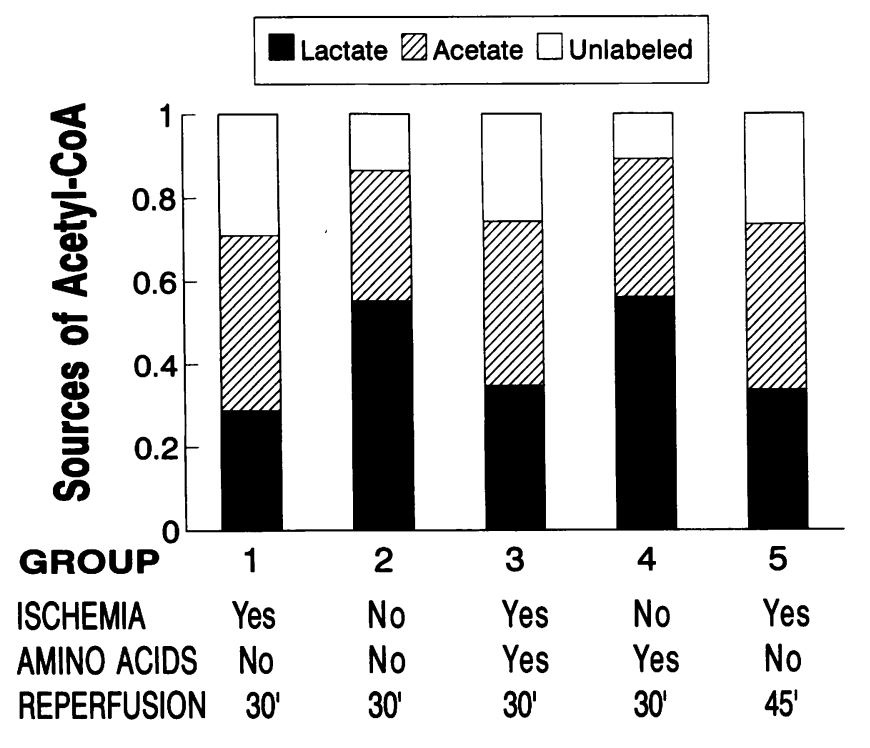

Figure 5. Sources of acetyl-CoA. The contribution of lactate, acetate, and unlabeled sources to acetyl-CoA measured by the nonsteady state analysis is shown for all groups.

anaplerotic pathway $(37,39)$. However, neither substrate selection by the myocardium nor anaplerotic flux were altered by the presence of glutamate and aspartate. These conclusions are based on measurements of anaplerosis and substrate selection when lactate and acetate metabolism are traced by ${ }^{13} \mathrm{C}$-NMR. Complementary studies with ${ }^{13} \mathrm{C}$-enriched glutamate or aspartate confirmed that neither amino acid is metabolized in the citric acid cycle under these conditions. Thus, our data do not support earlier suggestions that synthesis of tricarboxylic acid (TCA) cycle intermediates is stimulated or that glutamate and aspartate influence the selection of substrates for oxidation in the TCA cycle.

Anaplerosis occurred at a higher rate relative to TCA cycle flux after ischemia in this study. This determination depends on the assumption of metabolic steady state, a condition difficult to establish, but three observations indicate that the steady state analysis is valid. First, a method for measuring substrate selection that does not require steady state conditions yielded results that were similar to results obtained by the steady state analysis in both relevant groups. Second, substrate selection was not different in groups that were reperfused for different periods (groups 1 and 5, Table III), using the nonsteady state

Table IV. Flux through Anaplerotic Pathways Relative to Total Citric Acid Cycle Flux Calculated from the Steady State Analysis

\begin{tabular}{ll}
\hline Group & Anaplerosis \\
\hline 1, isc - & $0.20 \pm .04^{*}$ \\
2, nor - & $0.05 \pm .02$ \\
3, isc + & $0.20 \pm .04^{*}$ \\
4, nor + & $0.05 \pm .01$ \\
5, isc - & $0.14 \pm .03^{*}$
\end{tabular}

See Table I for abbreviations. Results are mean $\pm \operatorname{SD}(n=8)$.

* Different from groups 2 and 4 by analysis of variance, $P<0.05$.
A

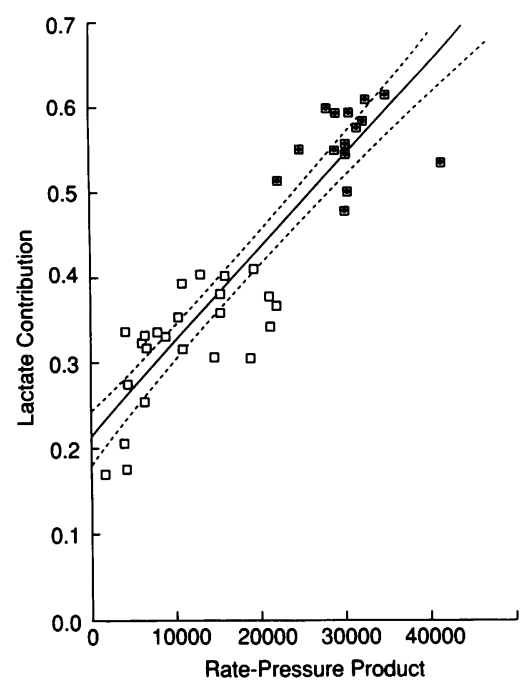

B
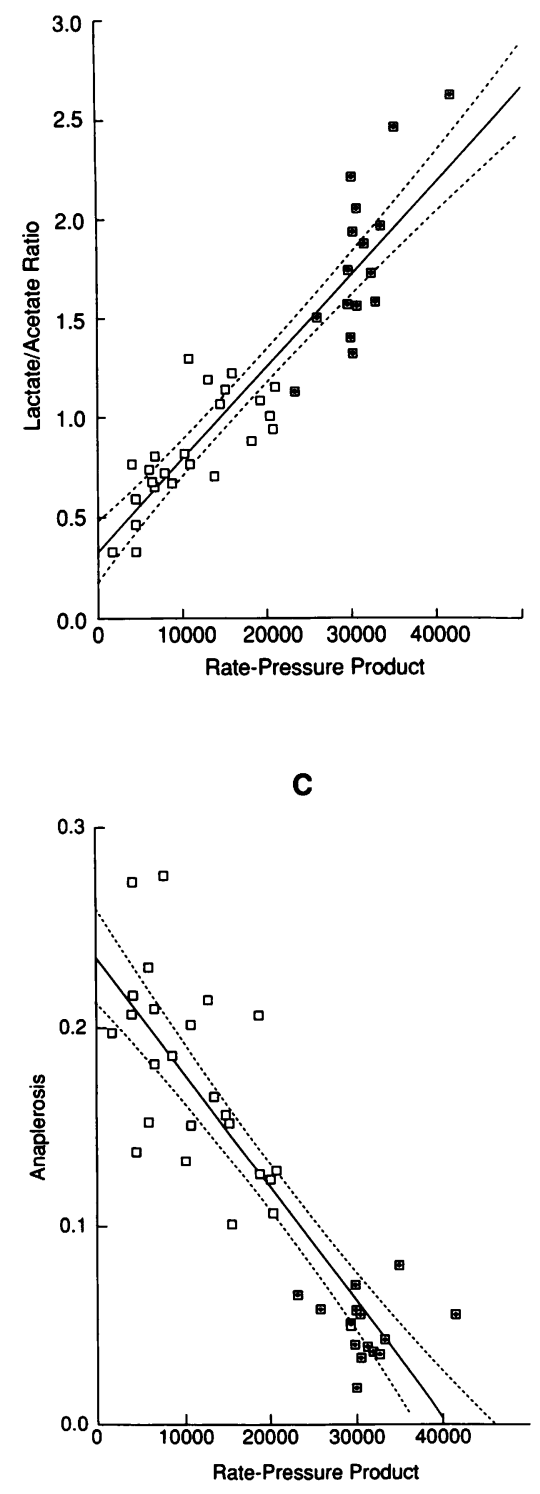

Figure 6. Relation between mechanical function and carbon flow into the citric acid cycle in reperfused myocardium. The rate-pressure product at the time of freeze clamping is compared with results of isotopomer analysis in ischemic ( $\square$ ) and nonischemic ( $\square)$ hearts. Linear regression and the $95 \%$ confidence intervals are shown for $A$, lactate contribution to acetyl-CoA; $B$, lactate/acetate utilization ratio; and $C$, anaplerosis. 
analysis. Finally, anaplerosis measured at either 30 or $45 \mathrm{~min}$ was substantially higher than control conditions.

The study is consistent with earlier work that found suppression of lactate relative to fatty acid (1) or acetate (40) utilization early during reperfusion. This effect has been attributed to suppression of pyruvate dehydrogenase activity during ischemia which effectively reduces carbohydrate contributions to acetyl-CoA. These data demonstrate a significant correlation between functional recovery after ischemia and substrate selection by the myocardium. Such information may prove useful in evaluating myocardial viability or in formulating strategies to improve cardiac performance after ischemia.

This study also illustrates the simplicity of analysis of substrate selection by a single ${ }^{13} \mathrm{C}-\mathrm{NMR}$ spectrum. Competition among two labeled substrates and unlabeled materials could be assessed in a single experiment without assuming metabolic or isotopic steady state. This method is generally useful in analysis of substrate selection during reperfusion where substrate selection is controversial.

\section{Acknowledgments}

We appreciate technical assistance by Angela Smiddy, Jacqueline Arenas, and Byron Franco and secretarial assistance by Lynne Owen and Debbie Shuttlesworth.

This study was supported by a Clinical Investigator Award and Merit Review of the Department of Veterans Affairs, National Institutes of Health grants HL34557, HL27472, and SCOR HL17669-17.

\section{References}

1. Mickle, D. A. G., P. J. del Nido, G. J. Wilson, R. D. Harding, and A. D. Romaschin. 1986. Exogenous substrate preference of the post-ischemic myocardium. Cardiovasc. Res. 20:256-263.

2. Schwaiger, M., R. A. Neese, L. Araujo, W. Wyns, J. A. Wisneski, H. Sochor, S. Swank, D. Kulber, C. Selin, M. Phelps, et al. 1989. Sustained nonoxidative glucose utilization and depletion of glycogen in reperfused canine myocardium. J. Am. Coll. Cardiol. 13:745-754.

3. Renstrom, B., S. H. Nellis, and A. J. Liedtke. 1990. Metabolic oxidation of pyruvate and lactate during early myocardial reperfusion. Circ. Res. 66:282-288

4. Myears, D. W., B. E. Sobel, and S. R. Bergmann. 1987. Substrate use in ischemic and reperfused canine myocardium: quantitative considerations. $\mathrm{Am}$. J. Physiol. 253:H107-H114.

5. Liedtke, A. J. 1981. Alterations of carbohydrate and lipid metabolism in the acutely ischemic heart. Prog. Cardiovasc. Dis. 23:321-336.

6. Lerch, R. A., H. D. Ambos, S. R. Bergmann, M. J. Welch, M. M. Ter-Pogossian, and B. E. Sobel. 1981. Localization of viable ischemic myocardium by positron emission tomography with "C palmitate. Circulation. 64:689-699.

7. Brown, M. A., D. W. Myears, and S. R. Bergmann. 1988. Noninvasive assessment of canine myocardial oxidative metabolism with carbon-11 acetate and positron emission tomography. J. Am. Coll. Cardiol. 12:1054-1063.

8. Brown, M. A., D. W. Myears, and S. R. Bergmann. 1989. Validity of estimates of myocardial oxidative metabolism with carbon-11 acetate and positron emission tomography despite altered patterns of substrate utilization. $J$. Nucl. Med. 30:187-193.

9. Armbrecht, J. J., D. B. Buxton, R. C. Brunken, M. E. Phelps, and H. R. Schelbert. 1989. Regional myocardial oxygen consumption determined noninvasively in humans with [ $\left.1-{ }^{11} \mathrm{C}\right]$ acetate and dynamic positron tomography. Circulation. 80:863-872.

10. Armbrecht, J. J., D. B. Buxton, and H. R. Schelbert. 1990. Validation of $\left[1-{ }^{11} \mathrm{C}\right]$ acetate as a tracer for noninvasive assessment of oxidative metabolism with positron emission tomography in normal, ischemic, postischemic, and hyperemic canine myocardium. Circulation. 81:1594-1605.

11. Buxton, D. B., C. A. Nienaber, A. Luxen, O. Ratib, H. Hansen, M. E. Phelps, and H. R. Schelbert. 1989. Noninvasive quantitation of regional myocardial oxygen consumption in vivo with $\left[1-{ }^{11} \mathrm{C}\right]$ acetate and dynamic positron emission tomography. Circulation. 79:134-142.

12. Lear, J. L. 1991. Relationship between myocardial clearance rates of carbon-11-acetate-derived radiolabel and oxidative metabolism: physiologic basis and clinical significance. J. Nucl. Med. 32:1957-1960.
13. Lazar, H. L., G. D. Buckberg, A. J. Manganaro, H. Becker, and J. V. Maloney, Jr. 1980. Reversal of ischemic damage with amino acid substrate enhancement during reperfusion. Surgery (St. Louis). 88:702-709.

14. Choong, Y. S., J. B. Gavin, and L. C. Armiger. 1988. Effects of glutamic acid on cardiac function and energy metabolism of rat heart during ischemia and reperfusion. J. Mol. Cell. Cardiol. 20:1043-1051.

15. Pisarenko, O. I., E. B. Novikova, L. I. Serebryakova, O. V. Tskitishvili, V. E. Ivanov, and I. M. Studneva. 1985. Function and metabolism of dog heart in ischemia and in subsequent reperfusion: effect of exogenous glutamic acid. Pfluegers Arch. Eur. J. Physiol. 405:377-383.

16. Bittl, J. A., and K. I. Shine. 1983. Protection of ischemic rabbit myocardium by glutamic acid. Am. J. Physiol. 245:H406-H412.

17. Bush, L. R., S. Warren, C. L. Mesh, and B. R. Lucchesi. 1981. Comparative effects of aspartate and glutamate during myocardial ischemia. Pharmacology (Basel). 23:297-304.

18. Rosenkranz, E. R., G. D. Buckberg, H. Laks, and D. G. Mulder. 1983. Warm induction of cardioplegia with glutamate enriched blood in coronary patients with cardiogenic shock who are dependent on inotropic drugs and intraaortic balloon support. J. Thorac. Cardiovasc. Surg. 86:507-518.

19. Yau, T. M., R. D. Weisel, D. A. G. Mickle, and J. Ivanov. 1992. Cardioplegia for the dysfunctional ventricle. In A Textbook of Cardioplegia for Difficult Clinical Problems. R. M. Engleman and S. Levitsky, editors. Futura Publishing Co., Inc. Mt. Kisco, NY. 89-91.

20. Pisarenko, O. I., O. D. Oleynikov, V. S. Shulzhenko, I. M. Studneva, I. M. Ryff, and V. I. Kapelko. 1989. Association of myocardial glutamate and aspartate pool and function recovery of postischemic heart. Biochem. Med. Metab. Biol. 42:105-117.

21. Challoner, D. R., and D. Steinberg. 1966. Effect of free fatty acid on the oxygen consumption of perfused rat heart. Am. J. Physiol. 210:280-286.

22. Vik-Mo, H., and O. D. Mjos. 1981. Influence of free fatty acids on myocardial oxygen consumption and ischemic injury. Am. J. Cardiol. 48:361-364.

23. Kjekshus, J. K., and D. Mjos. 1972. Effect of free fatty acids on myocardial function and metabolism in the ischemic dog heart. J. Clin. Invest. 51:17671776.

24. Bergman, G., L. Atkinson, J. Metcalfe, N. Jackson, and D. E. Jewitt. 1980. Beneficial effect of enhanced myocardial carbohydrate utilisation after oxfenicine (L-hydroxyphenyl-glycine) in angina pectoris. Eur. Heart J. 1:247-253.

25. Horowitz, J. D., S. T. B. Sia, P. S. MacDonald, A. J. Goble, and W. J. Louis. 1986. Perhexiline maleate treatment for severe angina pectoris-correlations with pharmacokinetics. Int. J. Cardiol. 13:219-229.

26. Cole, P. L., A. D. Beamer, N. McGowan, C. O. Cantillon, K. Benfell, R. A Kelly, L. H. Hartley, T. W. Smith, and E. M. Antman. 1990. Efficacy and safety of perhexiline maleate in refractory angina. A double-blind placebo-controlled clinical trial of a novel antianginal agent. Circulation. 81:1260-1270.

27. Wargovich, T. J., R. G. McDonald, J. A. Hill, R. L. Feldman, P. W. Stacpoole, and C. J. Pepine. 1988. Myocardial metabolic and hemodynamics effects of dichloroacetate in coronary artery disease. Am. J. Cardiol. 61:65-70.

28. Kornberg, H. L. 1966. Anaplerotic sequences and their role in metabolism. In Essays in Biochemistry. Vol 2. P. N. Campbell and R. D. Marshall, editors. Academic Press, London. 1-31.

29. Peuhkurinen K. S., T. E. S. Takala, E. M. Nuutinen, and I. E. Hassinen. 1983. Tricarboxylic acid cycle intermediates during ischemia in isolated perfused rat heart. Am. J. Physiol. 244:H281-H288.

30. Paulson, D. J., J. Traxler, M. Schmidt, J. Noonan, and A. L. Shug. 1986. Protection of the ischaemic myocardium by L-propionylcarnitine: effects on the recovery of cardiac output after ischaemia and reperfusion, carnitine transport and fatty acid oxidation. Cardiovasc. Res. 20:536-541.

31. Pisarenko, O. I., E. S. Solomatina, I. M. Studneva, V. E. Ivanov, V. I. Kapelko, and V. N. Smirnov. 1983. Effect of glutamic and aspartic acids on adenine nucleotides, nitrogenous compounds and contractile function during underperfusion of isolated rat heart. J. Mol. Cell. Cardiol. 15:53-60.

32. Peuhkurinen, K. J., E. M. Nuutinen, E. P. Pietilainen, J. K. Hiltunen, and I. E. Hassinen. 1982. Role of pyruvate carboxylation in the energy-linked regulation of pool sizes of tricarboxylic acid cycle intermediates in the myocardium. Biochem. J. 208:577-581.

33. Hoekenga, D. E., J. R. Brainard, and J. Y. Hutson. 1988. Rates of glycolysis and glycogenolysis during ischemia in glucose-insulin-potassium-treated perfused hearts: a ${ }^{13} \mathrm{C},{ }^{31} \mathrm{P}$ nuclear magnetic resonance study. Circ. Res. 62:10651074.

34. Shulman, G. I., L. Rossetti, D. L. Rothman, J. B. Blair, and D. Smith. 1987. Quantitative analysis of glycogen repletion by nuclear magnetic resonance spectroscopy in the conscious rat. J. Clin. Invest. 80:387-393.

35. London, R. E. $1988 .{ }^{13} \mathrm{C}$ labeling in studies of metabolic regulation. Prog. Nucl. Magn. Reson. Spect. 20:337-383.

36. Malloy, C. R., A. D. Sherry, and F. M. H. Jeffrey. 1988. Evaluation of carbon flux and substrate selection through alternate pathways involving the citric acid cycle of the heart by ${ }^{13} \mathrm{C}$ NMR spectroscopy. J. Biol. Chem. 263:69646971.

37. Malloy, C. R., A. D. Sherry, and F. M. H. Jeffrey. 1990. Analysis of 
tricarboxylic acid cycle of the heart using ${ }^{13} \mathrm{C}$ isotope isomers. Am. J. Physiol. 259:H987-H995.

38. Jeffrey, F. M. H., A. Rajagopal, C. R. Malloy, and A. D. Sherry. 1991. ${ }^{13} \mathrm{C}-\mathrm{NMR}$ : a simple yet comprehensive method for analysis of intermediary metabolism. Trends Biochem. Sci. 16:5-10.

39. Malloy, C. R. J. R. Thompson, F. M. H. Jeffrey, and A. D. Sherry. 1990. Contribution of exogenous substrates to acetyl coenzyme A: measurement by ${ }^{13} \mathrm{C}$ NMR under nonsteady-state conditions. Biochemistry. 29:6756-6761.

40. Sherry, A. D., C. R. Malloy, P. Zhao, and J. R. Thompson. 1992. Alterations in substrate utilization in the reperfused myocardium: a direct analysis by ${ }^{13} \mathrm{C}$ NMR. Biochemistry. 31:4833-4837.

41. Drake, A. J., J. R. Haines, and M. I. M. Noble. 1980. Preferential uptake of lactate by the normal myocardium in dogs. Cardiovasc. Res. 14:65-72.

42. Patel, T. B., and M. S. Olsen. 1984. Regulation of pyruvate dehydrogenase complex in ischemic rat heart. Am. J. Physiol. 246:H858-H864.

43. Guide for the Care and Use of Laboratory Animals, DHEW Publication No. (NIH) 85-23, Revised 1985, Office of Science and Health Reports, DRR/ NIH, Bethesda, MD.

44. Lowery, O. H., and J. V. Passonneau. 1972. A Flexible System of Enzymatic Analysis. Academic Press, New York.
45. SAS Institute Inc. 1985. SAS/STAT Guide for Personal Computers, version 6 edition. SAS Institute Inc., Cary, NC.

46. Galinanes, M., D. J. Chambers, and D. J. Hearse. 1992. Effect of sodium aspartate on the recovery of the rat heart from long-term hypothermic storage. $J$. Thorac. Cardiovasc. Surg. 103:521-531.

47. Haas, G. S., L. W. V. DeBoer, D. D. O'Keefe, R. M. Bodenhamer, G. A. Geffin, L. J. Drop, R. S. Teplick, and W. M. Daggett. 1984. Circulation. 70(Suppl I):I-65-I-74.

48. Thomassen, A., T. T. Nielsen, J. P. Bagger, and P. Henningsen. 1991. Effects of intravenous glutamate on substrate availability and utilization across the human heart and leg. Metab. Clin. Exp. 40:378-389.

49. Thomassen, A. T. T. Nielsen, J. P. Bagger, A. K. Pedersen, and P. Henningsen. 1991. Antiischemic and metabolic effects of glutamate during pacing in patients with stable angina pectoris secondary to either coronary artery disease or syndrome X. Am. J. Cardiol. 68:291-295.

50. Scrutton, M. C., and M. D. White. 1974. Pyruvate carboxylase: inhibition of the mammalian and avian liver enzymes by $\alpha$-ketoglutarate and L-glutamate. J. Biol. Chem. 249:5405-5415.

51. Peuhkurinen, K. J. 1984. Regulation of the tricarboxylic acid cycle pool size in heart muscle. J. Mol. Cell. Cardiol. 16:487-495. 